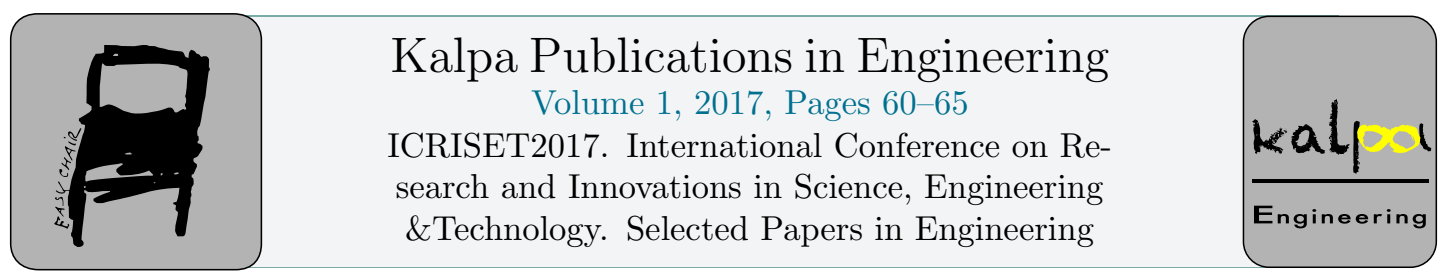

\title{
Design and Analysis of Force/Moment Sensor for a Robot
}

\author{
Kenalkumar D. Tandel ${ }^{1}$, Dr. Haresh P. Patolia ${ }^{2}$ and Dr. Vinay J Patel ${ }^{3}$ \\ ${ }^{1} \mathrm{M}$. Tech Machine Design, ${ }^{2}$ Associate Professor, ${ }^{3}$ Professor \\ ${ }^{1,2,3}$ Mechanical Engineering Department, BVM Engineering College, V V Nagar, Anand, India \\ ${ }^{1}$ tandelkenal10@gmail.com, ${ }^{2}$ hppatolia@bvmengineering.ac.in, \\ ${ }^{3}$ vkjp1966@gmail.com
}

\begin{abstract}
To safely hold an unidentified object by means of an intelligent hand of robot, the hand has to recognize the weight of it. By attaching six-axis Force/Torque ( $\mathrm{F} / \mathrm{T}$ or Force/Moment) sensor to an intelligent robot's hand the weight can be calculated by measuring forces $F_{x}, F_{y}$ and $F_{z}$. Forces should be measured in order to precisely pull and push an object. To securely grasp an unidentified object with an intelligent robot's gripper, the forces in the gripping direction and in the gravitational direction needs to be detected, but it also requires to perceive the moments to accurately recognize the position of the object in the grippers. A robot joint can be controlled in better way if three forces and three moments exerted at the joint are measured. The available Force/Torque sensors are bulky, not customized and costly. Therefore, it is essential to customize and develop low cost six axis Force/Torque sensor with new appropriate dimensions for an intelligent robot's joints. Six axis Force/Torque sensor is designed using strain gauge. The strain gauges are selected for Aluminium and its working conditions. The sensor design is based on results of parametric analysis done in ANSYS software to obtain the strain values in the measurable range. The analytical results are compared with Finite Element Analysis (ANSYS) results. The percentage error in deviation is $0.75 \%$ maximum.
\end{abstract}

Keywords: Sensor, Force/Torque sensor, Force measurement, intelligent robot.

\section{INTRODUCTION}

The intelligent robot hand is required to recognize the weight of the object to be picked safely by it. The weight of object can be calculated by measuring forces $F x, F y$ and $F z$ with the help of six-axis Force/Moment sensor attached to the intelligent robot wrist. The forces in the gripping direction and in the gravitational direction needs to perceive with an intelligent robot gripper to grasp an unidentified object securely. However, griping is also required to perceive the moments about all three axes accurately to identify the position and orientation of the object in the grippers. The force sensing at each joint also helps to control the joint in better way while pushing or pulling the unidentified object. Force and moment perception at each joint of biped system helps to control the joints of biped system while walking. In general, a robot joint can be controlled in better way if forces and moments exerted at the joints are perceived precisely [1]. 
Numerous multi-dimensional force sensors have been established in the previous decades, which are planned for use at the end effector and/or joints of a robot to observer force and/or moment. GabSoon Kim et al have designed and fabricated the structure of three axis Force/Torque sensors using plate-beams [2]. The sensor may be used for sensing the forces along $\mathrm{x}$-axis, $\mathrm{y}$-axis and torque about $\mathrm{z}$ axis. The use of sensor is limited to the joints of industrial robots. The work is extended to develop and fabricate a six-axis Force/Torque sensor [3]. The analytical analysis and FEA analysis of strain is compared with experimental results. It is found that interference error is less than $2 \%$. This sensor is also limited to the joints of industrial robots due to its complexity. A structure of small capacity sixaxis Force/Torque sensor is modelled, designed and manufactured by G. Kim [4] to determine the uncertainty of the sensor. The sensor is evaluated for the uncertainty using suggested calibration method. The results confirm the uncertainty of the sensor less than $2.78 \%$.

Multi-fingered robot hands with rigid or soft hemispheric finger ends require intelligent control for grasping and manipulating of an object [5]. It also further reveals that simplified feedback sensors are required without object kinematics and location of the mass center. A strain gauge based gripper for intelligent robot to measure three forces and moments is developed which gives less than $6 \%$ maximum error of the rated strain [6]. This sensor gives better control for intelligent task with less than $2.79 \%$ maximum interference error. The work is extended to measure forces and torques at finger with less than $9.7 \%$ maximum error of rated strain and less than $3.93 \%$ maximum interference error [7].

G. Kim has modified model of plate beam six-axis force/torque sensor using rectangular taper beams for an intelligent robot to increase the sensitivity of the sensor without disturbing its rigidity [8]. This sensor can be used to accurately push and pull an object with an intelligent robot's hand and in order to safely walk with an intelligent robot's foot. The designed sensor was analyzed by FEM and fabricated similar to size of human being wrist i.e. diameter about $60-80 \mathrm{~mm}$ and the thickness about 20-40 $\mathrm{mm}$ [9]. The rated outputs from the derived equations and FEM analysis reported well to those from the experiments with interference error of the sensor less than 2.8\%. Dan Feng Chen et al have designed and calibrated a six-axis force/torque sensor for large measurement range used in the space manipulator [10].

Many of the above literature have reported analytical analysis and FEM analysis to model the sensor with full bridge strain gauge arrangement. The most of the sensors are not compact and complex in fabrication. In this paper, the compact and simple design of force/torque sensor using full bridge strain gauge sensor is presented for wrist joint of robot hand and knee joint of leg system. The paper follows as: section 2 describes design of the sensor, section 3 presents analytical analysis of the sensor, in section 4 the results are compared and finally section 5 concludes the work.

\section{DESIGN OF THE SENSOR}

To measure force and torque simultaneously for typical wrist and knee joints, basic model can be proposed which consist of outer ring, inner transfer ring and four flexible arm (beams) of aluminum. Figure 1 shows the basic drawing of six axis Force/Torque sensor for wrist and knee joint with positions of strain gauges. Different dimensions for wrist and knee joint are tabulated in Table 1 .

\begin{tabular}{|c|c|c|c|c|c|c|}
\hline \multirow{2}{*}{$\begin{array}{c}\text { Type of } \\
\text { sensor }\end{array}$} & \begin{tabular}{c}
$\mathbf{D 1}$ \\
\cline { 2 - 6 }
\end{tabular} & $\begin{array}{c}\mathbf{D 2} \\
(\mathbf{m m})\end{array}$ & $\begin{array}{c}\mathbf{D 3} \\
(\mathbf{m m})\end{array}$ & $\begin{array}{c}\mathbf{D 4} \\
(\mathbf{m m})\end{array}$ & $\begin{array}{c}\mathbf{D P 1} \\
(\mathbf{m m})\end{array}$ & $\begin{array}{c}\mathbf{D P 2} \\
(\mathbf{m m})\end{array}$ \\
\hline $\begin{array}{c}\text { Wrist } \\
\text { sensor }\end{array}$ & 85 & 75 & 38 & 20 & 80 & 30 \\
\hline
\end{tabular}
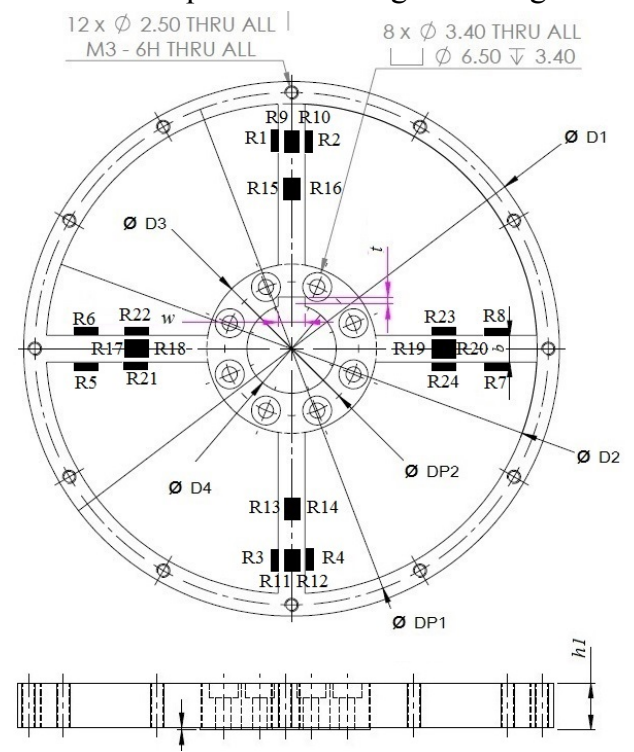

Figure 1: Basic drawing of six axis Force/Torque sensor for wrist and knee with strain gauges 


\begin{tabular}{|l|l|l|l|l|l|l|}
\hline \multirow{2}{*}{$\begin{array}{c}\text { Type of } \\
\text { sensor }\end{array}$} & \multicolumn{7}{|c|}{ Variable dimensions } \\
\cline { 2 - 7 } & $\begin{array}{c}\mathbf{D 1} \boldsymbol{\mathbf { m }}) \\
(\mathbf{m m})\end{array}$ & $\begin{array}{c}\mathbf{D 3} \\
(\mathbf{m m})\end{array}$ & $\begin{array}{c}\mathbf{D 4} \\
(\mathbf{m m})\end{array}$ & $\begin{array}{c}\mathbf{D P 1} \\
(\mathbf{m m})\end{array}$ & $\begin{array}{c}\mathbf{D P 2} \\
(\mathbf{m m})\end{array}$ \\
\hline $\begin{array}{l}\text { Knee } \\
\text { sensor }\end{array}$ & 120 & 110 & 38 & 20 & 115 & 30 \\
\hline
\end{tabular}

Table 1: Dimensions For Different Sensors

For both the sensors values of $\mathrm{w}, \mathrm{t}, \mathrm{b}, \mathrm{h} 1 \& \mathrm{~h} 2$ are $6 \mathrm{~mm}, 1.6 \mathrm{~mm}, 6 \mathrm{~mm}, 10.5 \mathrm{~mm} \& 0.5 \mathrm{~mm}$ respectively. For measurement of force $F x$, strain gauges $\mathrm{R} 1 \sim \mathrm{R} 4$ are provided. Similarly, for measurement of forces $F y, F z$ and moments $M x, M y$ and $M z$, strain gauges R5 R8, R9 R12, R13 R16, R17 R20 and R21 R24 are provided respectively. The strain gauges are associated into a Wheatstone Bridge circuit with an arrangement of four active gauges to make it full bridge circuit. Strain gauges available now can give the measurable value of output for small strain values, like for strain values of order $10^{-6}$ can produce measurable output voltages which will make sensor more compact.

\section{ANALYTICAL ANALYSIS OF F/T SENSOR}

F/T sensor is made up of 4 beams (A, B, C \& D) of equal size. Dimensions of beams are length $=l$, height $=h$ and width $=b$ as in following Figure 1 .

On the base of symmetricity and the set of equations required for forces and moments, it is observed that the equation of force $F x$ can also be used for $F y$ and similarly for moments $M x$ and $M y$.

To determine the values of strains due to forces $F x, F y$ and $F z$ and moments $M x, M y$ and $M z$ analytically the following four cases can be referred:

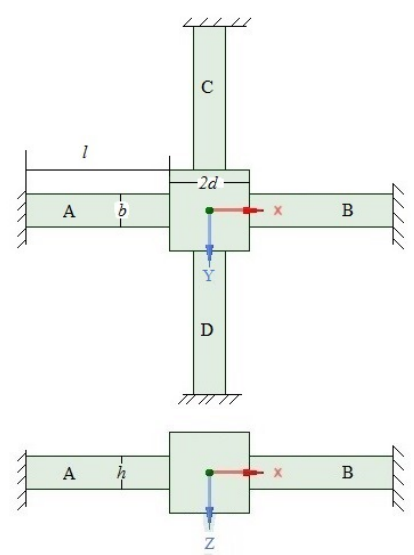

(A)

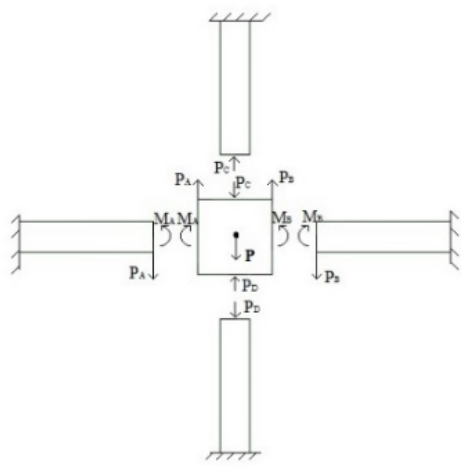

(B)

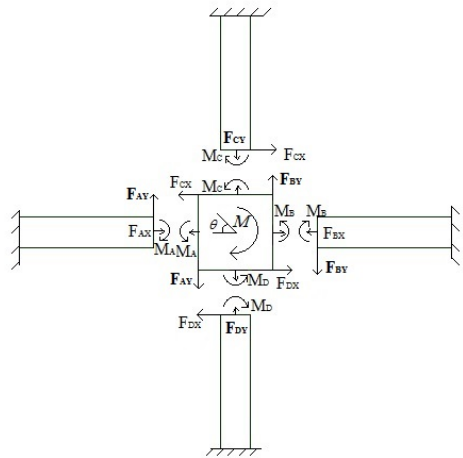

(C)

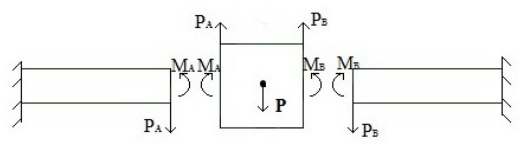

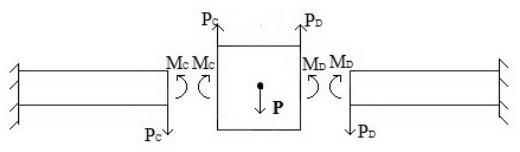

(D)

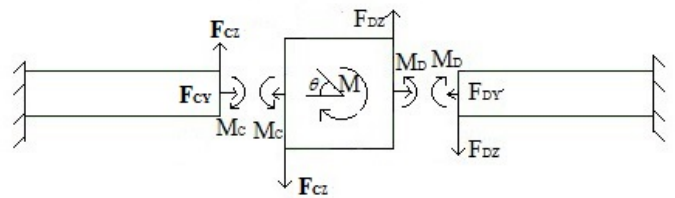

(E)

Figure 2: F.B.D when(A) no load (B) $F_{x}=P N$, (C) $M_{Z}=M$ Nm (D) $F_{Z}=P$ N and (E) $M_{x}=M N m$ 


\subsection{Calculation for measuring strain due to force $F_{x}\left(\right.$ or $\left.F_{y}\right)=P \mathrm{~N}$.}

From Figure $2(\mathrm{~B})$, it can be seen that beam $\mathrm{A}$ and $\mathrm{B}$ are under bending and beam $\mathrm{C}$ and $\mathrm{D}$ are under axial loading. So $P=P_{A}+P_{B}+P_{C}+P_{D}=\frac{A E \delta}{l}+\frac{A E \delta}{l}+\frac{3 E I_{Y} \delta}{l^{3}}+\frac{3 E I_{Y} \delta}{l^{3}}$

$$
\delta=\left[\frac{P}{\frac{2 A E}{l}+\frac{6 E I_{Y}}{l^{3}}}\right]
$$

Now for beam A, $P_{A}=\left[\frac{3 E I_{Y} \delta}{l^{3}}\right]$, Put the $\delta$ from (1), $P_{A}=\left[\frac{3 P I_{Y}}{2 A l^{2}+6 I_{Y}}\right]$

Moment $M_{A}$ at the end of beam A can be written as, $M_{A}=P_{A} \times \frac{l}{2}=\left[\frac{3 P I_{Y}}{2 A l^{2}+6 I_{Y}}\right] \times \frac{l}{2}$

Moment at arbitrary point $x$ leads to, $M_{A}=\left[\frac{3 P I_{Z}}{2 A l^{2}+6 I_{Y}}\right] \times\left(x-\frac{l}{2}\right)$

From Hook's law, $\sigma=\varepsilon E$ and From Bending equation, $\sigma=\frac{M y}{I}$

So, $\varepsilon E=\frac{M_{A} y}{I_{Y}}=\frac{M_{A} y}{I_{Y} E}=\frac{M_{A}}{E Z_{P Y}} \quad\left(\because Z_{P Y}=\frac{I_{Y}}{y}\right)$

From (2) and (3) strain on upper and lower part of beam is given as,

$$
\varepsilon_{U}=\left[\frac{3 P I_{Y}}{2 A l^{2}+6 I_{Y}}\right] \times\left(x-\frac{l}{2}\right) \times \frac{1}{E Z_{P Y}} \text { and } \varepsilon_{L}=\left[\frac{-3 P I_{Y}}{2 A l^{2}+6 I_{Y}}\right] \times\left(x-\frac{l}{2}\right) \times \frac{1}{E Z_{P Y}}
$$

\subsection{Calculation for measuring strain due to force $F_{z}=P \mathrm{~N}$.}

From Figure 2(D), it is observed that beam A, B, C and D are under bending. Under the effect of applied force $P$, the produced reaction force $P_{A}, P_{B}, P_{C}$ and $P_{D}$ can be represented as,

$$
P=P_{A}+P_{B}+P_{C}+P_{D}=\frac{3 E I_{Z} \delta}{l^{3}}+\frac{3 E I_{Z} \delta}{l^{3}}+\frac{3 E I_{Z} \delta}{l^{3}}+\frac{3 E I_{Z} \delta}{l^{3}}
$$

Similar to previous case for beam $\mathrm{C}$, strain on upper and lower part of beam is given as,

$$
\varepsilon_{U}=\left[\frac{P}{4}\right] \times\left(y-\frac{l}{2}\right) \times \frac{1}{E Z_{P Z}} \text { and } \varepsilon_{L}=\left[\frac{-P}{4}\right] \times\left(y-\frac{l}{2}\right) \times \frac{1}{E Z_{P Z}}
$$

\subsection{Calculation for measuring strain due to moment $M_{x}$ (or $\left.M y\right)=M \mathrm{Nm}$.}

Figure 2(C), shows free body diagram when moment $\mathrm{M} \mathrm{Nm}$ applied about $\mathrm{X}$ axis. Reaction generated due to application of moment are represented in the same.

Moment $M_{C}$ at the end of beam $\mathrm{C}$ can be written as, $M_{C}=\frac{M_{x}}{2}$ 
The forces $F_{C Y}, F_{C Z}$ and moment $M_{C}$ as referred in [3] can be written as, $F_{C Y}=\frac{A E d \theta^{2}}{l}$, $F_{C Z}=\frac{12 E I_{Z} \theta}{l^{3}}\left(d+\frac{l}{2}\right)$ and $M_{C}=\frac{12 E I_{Z} \theta}{l^{2}}\left(\frac{d}{2}+\frac{l}{3}\right)$

By using moment equilibrium condition, $\frac{M_{x}}{2}=d F_{C Z}+M_{C}$

From (6) and (7) $\theta=\frac{M_{x} / 2}{\left[\frac{12 E I_{Z} d}{l^{3}}\left(d+\frac{l}{2}\right)+\frac{12 E I_{Z}}{l^{2}}\left(\frac{d}{2}+\frac{l}{3}\right)\right]}$

Moment at arbitrary point $y$ leads to, $M_{C^{\prime}}=M_{C}-y F_{C Z}=\frac{12 E I_{Z} \theta}{l^{2}}\left[d\left(\frac{1}{2}-\frac{y}{l}\right)+l\left(\frac{1}{3}-\frac{y}{2 l}\right)\right]$

The equations for analysing the strains on the upper and lower part of plate-beam $\mathrm{C}$ can be developed by uniting the strain developed due to tension and bending, can be expressed as,

$$
\varepsilon_{U}=\frac{6 h \theta}{l^{2}}\left[d\left(\frac{1}{2}-\frac{y}{l}\right)+l\left(\frac{1}{3}-\frac{y}{2 l}\right)\right]+\frac{d \theta^{2}}{l} \text { and } \varepsilon_{L}=\frac{-6 h \theta}{l^{2}}\left[d\left(\frac{1}{2}-\frac{y}{l}\right)+l\left(\frac{1}{3}-\frac{y}{2 l}\right)\right]+\frac{d \theta^{2}}{l}
$$

\subsection{Calculation for measuring strain due to moment $M_{Z}=M \mathrm{Nm}$.}

Figure 2(E), indicates reaction forces and moments generated at the end of beams A, B, C and D due to moment $M \mathrm{Nm}$ applied about $\mathrm{Z}$ axis.

Moment $M_{A}$ at the end of beam A can be written as, $M_{A}=\frac{M_{Z}}{4}$

As in previous case we can find $\theta=\frac{M_{Z} / 4}{\left[\frac{12 E I_{Y} d}{l^{3}}\left(d+\frac{l}{2}\right)+\frac{12 E I_{Y}}{l^{2}}\left(\frac{d}{2}+\frac{l}{3}\right)\right]}$ and equation for analysing the strains on the upper and lower part of plate-beam A can be developed by uniting the tension strain and the bending strain, which are written as,

$$
\varepsilon_{U}=\frac{6 b \theta}{l^{2}}\left[d\left(\frac{1}{2}-\frac{x}{l}\right)+l\left(\frac{1}{3}-\frac{x}{2 l}\right)\right]+\frac{d \theta^{2}}{l} \text { and } \varepsilon_{L}=\frac{-6 b \theta}{l^{2}}\left[d\left(\frac{1}{2}-\frac{x}{l}\right)+l\left(\frac{1}{3}-\frac{x}{2 l}\right)\right]+\frac{d \theta^{2}}{l}
$$

Equations (4), (5), (9), (10) and (11)can be used to estimate strain in the beams at particular point from inner ring end on beam when Force and/or Moment is apply on transfer ring, where $A=b \times h$, $I_{Y}=\frac{h b^{3}}{12}, Z_{P Y}=\frac{h b^{2}}{6}, I_{Z}=\frac{b h^{3}}{12}$ and $Z_{P Z}=\frac{b h^{2}}{6}$. These set of equations also can be used to estimate force and/or torque produced for a known values of strain applied.

\section{COMPARISON BETWEEN STRAIN MEASURED BY ANALYTICAL AND ANSYS}

By applying different forces and moments, strain values are found out using analytical equations derived in section III and using ANSYS. Strain values found out by both methods are compared and \% error is calculated. Results of calculation are tabulated in Table 2. 


\begin{tabular}{|c|c|c|c|}
\hline \multirow{2}{*}{ Parameters } & \multicolumn{2}{|c|}{ Strain calculation methods } & \multirow{2}{*}{ Error \% } \\
\cline { 2 - 3 } & Strain in ANSYS & Strain by equations & \multirow{2}{*}{$0.60 \%$} \\
\hline $\begin{array}{c}F y=120 \mathrm{~N} \\
x=12 \mathrm{~mm}\end{array}$ & $9.8644 \times 10^{-7}$ & $9.9243 \times 10^{-7}$ & $0.51 \%$ \\
\hline $\begin{array}{c}F z=120 \mathrm{~N} \\
y=12 \mathrm{~mm}\end{array}$ & $1.1679 \times 10^{-5}$ & $1.1620 \times 10^{-5}$ & $0.75 \%$ \\
\hline $\begin{array}{c}M x=48 \mathrm{Nmm} \\
\mathrm{y}=(-12) \mathrm{mm}\end{array}$ & $1.6627 \times 10^{-7}$ & $1.6755 \times 10^{-7}$ & $0.56 \%$ \\
\hline $\begin{array}{c}M z=48 \mathrm{Nmm} \\
\mathrm{x}=(-12) \mathrm{mm}\end{array}$ & $1.0250 \times 10^{-7}$ & $1.0193 \times 10^{-7}$ & \multirow{2}{*}{} \\
\hline
\end{tabular}

Table 2: Comparison Between ANSYS And Analytical

\section{CONCLUSION}

The sensor design is based on results of parametric analysis done in ANSYS software to obtain the strain values in the measurable range. The FEA (ANSYS) results are compared with analytical results. The percentage error in deviation is $0.73 \%$ maximum. So it allows to use derived analytical equations for further optimization of design and if strain generated due to Force/Torque in beam can be found out by any means (using strain gauges) these equations can also be used for finding the force and/or torque value. The six-axis Force/Torque sensor can also be optimized with the help of analytical equations derived for six axis $\mathrm{F} / \mathrm{T}$ sensor. These equations can also be verified by performing experimental strain analysis of six-axis Force/Torque and can be compared with the analytical analysis and FEM analysis.

\section{REFERENCES}

[1] C. Xiong, Y. Li, Y. Xiong, H. Ding and Q. Huang, "Grasp capability analysis of mutifingered robot hands", Rbotics and Autonomous Systems, vol. 27, pp. 211-224, 1999.

[2] G.-S. Kim, D.-I. Kang, K.-W. Um and S.-H. Rhee, "Design and fabrication of three-component force/moment sensor using plate-beams", Measurement Science and Technology, vol. 10, pp. 295-301, 1999.

[3] G.-S. Kim, D.-I. Kang and S.-H. Rhee, "Design and fabrication of a six-component force/moment sensor", Sensors and Actuators, vol. 77, pp. 209-220, 1999.

[4] G.-S. Kim, "The design of six-component force/moment sensor and evaluation of its uncertainty", Measurement Science and Technology, vol. 12, pp. 1445-1455, 2001.

[5] S. Arimoto, "Intelligent control of multi-fingered hands," Annual Revies in control, vol. 28, pp. 75-85, 2008.

[6] G.-S. Kim and H.-D. Lee, "Development of a six-axis force/moment sensor and its control system for an intelligent robot's finger", Measurement Science and Technology, vol. 14, pp. 1265-1274, 2003.

[7] G.-S. Kim, "Development of a small 6-axis force/moment sensor for robot's fingers", Mesurement Science and Technology, vol. 15, pp. 2233-2238, 2004.

[8] G.-S. Kim, "Developmetn of a six-axis force/moment sensor with rectangular taper beams for an intelligent robot", International journal of control, automation, and systems, vol. 5, no. 4, pp. 419-428, 2007.

[9] G.-S. Kim, "Design of a six-axis wrist force/moment sensor using FEM and its fabrication for an intelligent robot", Sensors and Actuators, vol. 133, pp. 27-34, 2007.

[10] D. Chen, A. Song and A. Li, "Design and calibration of a six-axis force/torque sensor with large measurement range used for the space manipulator", Procedia Engineering, vol. 99, pp. 1164$1170,2015$. 